Preprint typeset using IATEX style AASTeX6 v. 1.0

\title{
CUMULATIVE NEUTRINO AND GAMMA-RAY BACKGROUNDS FROM HALO AND GALAXY MERGERS
}

\author{
Chengchao Yuan ${ }^{1}$, Peter Mészáros ${ }^{1}$, Kohta Murase $^{1,2}$, and Donghui Jeong ${ }^{1}$
}

\footnotetext{
${ }^{1}$ Department of Physics; Department of Astronomy and Astrophysics; Center for Particle and Gravitational Astrophysics, The Pennsylvania State University, University Park, PA 16802, USA

${ }^{2}$ Center for Gravitational Physics, Yukawa Institute for Theoretical Physics, Kyoto University, Kyoto 606-8502, Japan
}

\section{ABSTRACT}

The merger of dark matter halos and the gaseous structures embedded in them, such as proto-galaxies, galaxies, and groups and clusters of galaxies, results in strong shocks that are capable of accelerating cosmic rays $(\mathrm{CRs})$ to $\gtrsim 10 \mathrm{PeV}$. These shocks will produce high-energy neutrinos and $\gamma$-rays through inelastic $p p$ collisions. In this work, we study the contributions of these halo mergers to the diffuse neutrino flux and to the non-blazar portion of the extragalactic $\gamma$-ray background. We formulate the redshift dependence of the shock velocity, galactic radius, halo gas content and galactic/intergalactic magnetic fields over the dark matter halo distribution up to a redshift $z=10$. We find that high-redshift mergers contribute a significant amount of the cosmic-ray luminosity density, and the resulting neutrino spectra could explain a large part of the observed diffuse neutrino flux above $0.1 \mathrm{PeV}$ up to $\sim \mathrm{PeV}$. We also show that our model can somewhat alleviate tensions with the extragalactic $\gamma$-ray background. First, since a larger fraction of the CR luminosity density comes from high redshifts, the accompanying $\gamma$-rays are more strongly suppressed through $\gamma \gamma$ annihilations with the cosmic microwave background (CMB) and the extragalactic background light (EBL). Second, mildly radiative-cooled shocks may lead to a harder CR spectrum with spectral indices of $1.5 \lesssim s \lesssim 2.0$. Our study suggests that halo mergers, a fraction of which may also induce starbursts in the merged galaxies, can be promising neutrino emitters without violating the existing Fermi $\gamma$-ray constraints on the non-blazar component of the extragalactic $\gamma$-ray background.

Keywords: cosmic rays — galaxies: halos — galaxies: clusters: general — neutrinos — gamma rays: diffuse background

\section{INTRODUCTION}

Neutrino astrophysics has made substantial progress since the IceCube Neutrino Observatory in Antarctic (e.g., Gaisser \& Halzen 2014; Halzen 2016, for reviews) (hereafter, IceCube) was completed. During the last half decade, scores of high-energy (HE) astrophysical neutrinos with energies between $\sim 10 \mathrm{TeV}$ and a few $\mathrm{PeV}$ have been detected by IceCube, and the number keeps growing (Aartsen et al. 2013a,b, 2014, 2015). The arrival directions of these neutrinos are compatible with an isotropic distribution even in the $10-100 \mathrm{TeV}$ range, suggesting that a large part of these diffuse neutrinos come from extragalactic sources. Non-observation of diffuse Galactic $\gamma$-rays from the Galactic plane and other extended regions independently suggest that the Galactic contribution (e.g., by Fermi bubbles or local supernova remnants) is unlikely to be dominant (Ahlers \& Murase 2014;
Apel et al. 2017; Abeysekara et al. 2017a,b). However, despite extensive efforts, the physical nature of the sources of the diffuse neutrinos still remains in dispute. Possible candidates include gamma-ray bursts (GRBs) (e.g., Waxman \& Bahcall 1997; Meszaros \& Waxman 2001; Murase 2008; Wang \& Dai 2009; Baerwald et al. 2013; Bustamante et al. 2014; Tamborra \& Ando 2016), low-power GRBs (Murase et al. 2006; Gupta \& Zhang 2007; Murase \& Ioka 2013; Xiao \& Dai 2014, 2015; Senno et al. 2016; Denton \& Tamborra 2017), radioloud active galactic nuclei (AGNs) (e.g., Mannheim 1995; Halzen \& Zas 1997; Anchordoqui et al. 2008; Murase et al. 2014; Dermer et al. 2014; Becker Tjus et al. 2014; Petropoulou et al. 2015; Padovani et al. 2015; Blanco \& Hooper 2017), radioquiet/low-luminosity AGNs (Stecker et al. 1991; Alvarez-Muñiz \& Mészáros 2004; Stecker 2013; Kimura et al. 2015), and AGNs embedded in galaxy 
clusters and groups ${ }^{1}$. It is generally accepted that the bulk of astrophysical neutrinos are generated by charged pion $\left(\pi^{ \pm}\right)$decays, and that these pions are the secondaries from cosmic ray $(\mathrm{CR})$ particles undergoing hadronuclear $(p p)$ or photohadronic $(p \gamma)$ interactions between the CRs and ambient target gas nuclei or photons. Meanwhile, these collisions also lead unavoidably to neutral pions $\left(\pi^{0}\right)$ as well, which subsequently decay into a pair of $\gamma$-rays. Hence, the diffuse neutrino flux is expected to have an intimate connection with the diffuse CR and $\gamma$-ray backgrounds, and multi-messenger analyses need to be applied to constrain the origin of these diffuse high-energy cosmic particle fluxes (Murase et al. 2013, 2016; Bechtol et al. 2017).

Galaxy clusters and groups have been considered as promising candidate sources of IceCube's neutrinos, and CR accelerators can be not only AGNs but also intragalactic sources, accretion shocks, and mergers of clusters and groups (e.g., Murase et al. 2008; Fang \& Murase 2018). Star-forming and starburst galaxies (SFGs \& SBGs, respectively) have also been suggested as promising candidates for HE neutrino sources (e.g., Loeb \& Waxman 2006; Murase et al. 2013; Tamborra et al. 2014; Anchordoqui et al. 2014; Chang \& Wang 2014; Chang et al. 2015; Senno et al. 2015; Chakraborty \& Izaguirre 2015). In particular, starburst galaxies have dense gaseous environments and have been of interest as efficient CR reservoirs. Previous studies have assumed not only supernova and hypernova remnants (SNRs \& HNRs, respectively) but also galaxy mergers, disk-driven outflows and possible weak jets from AGNs as CR accelerators embedded in the starforming galaxies (Murase et al. 2013; Tamborra et al. 2014; Kashiyama \& Mészáros 2014; Senno et al. 2015; Chakraborty \& Izaguirre 2015; Lamastra et al. 2017). Hypernovae (HNe) are a subclass of Type $\mathrm{Ib} / \mathrm{c}$ supernovae (SNe), essentially a hyper-energetic version of $\mathrm{Ib} / \mathrm{c}$ SNe. The typical ejecta energy of $\mathrm{HNe}$ is $10^{52} \mathrm{erg}$, which is one order of magnitude larger than for SNe. Like SNRs, a hypernova remnant (HNR) leads to an extended structure that results from a hypernova explosion. In any case, an important constraint on such models is provided by the extragalactic $\gamma$-ray background (EGB) in the $100 \mathrm{MeV}-820 \mathrm{GeV}$ range, derived from the observation by the Fermi-LAT satellite (Ackermann et al. 2015). Recent studies of the blazar flux distribution at $\gamma$-ray energies above $50 \mathrm{GeV}$ indicate that blazars account for $86_{-14}^{+16 \%}$ of the total EGB flux (Ackermann et al. 2016). This provides a strong

\footnotetext{
${ }^{1}$ Groups of galaxies are smaller clusters, numbering from a few to dozens of galaxies.
}

constraint, namely, only a fraction $\lesssim 30 \%$, with a best fit of $14 \%$, can be ascribed to any remaining non-blazar component of the EGB (see also Lisanti et al. 2016).

With this constraint, the SBG scenario is apparently disfavored as the dominant origin of IceCube neutrinos (Bechtol et al. 2017). However, so far, this conclusion depends on the interpretation of the mediumenergy neutrino data in the $10-100 \mathrm{TeV}$ range. For example, the cumulative neutrino background may consist of two components, in which the high-energy data above $\sim 100 \mathrm{TeV}$ can be explained by the SBGs. On the other hand, the $10-100 \mathrm{TeV}$ component motivates CR accelerators that are "dark" in $\gamma$-rays (Murase et al. 2016) to satisfy multi-messenger constraints. The $\gamma$-rays may be attenuated inside their sources, or they might be absorbed during the propagation. Possible candidates include choked-jet GRBs or high-redshift sources such as Pop-III HNRs embedded in starbursts (Xiao et al. 2016).

In this paper, we focus on halo mergers as an origin of $\mathrm{HE}$ neutrinos. In the standard hierarchical galaxy formation scenario, galaxies form inside extended dark matter halos. When dark matter halos merge, the galaxies in these halos also merge, and the collision of the cold gas in the merging galaxies leads to shocks on a galactic scale in the galactic interstellar medium (ISM) gas. Later in the process of cosmological structure formation, at lower redshifts where galaxy groups and galaxy clusters have started forming, mergers among the dark matter halos containing these groups and clusters are also expected, These mergers are very energetic and result in shocks in the intergalactic medium (IGM) gas of the participating groups/clusters. One vivid example is the Bullet Cluster (Clowe et al. 2004; Markevitch et al. 2004). Both these galactic and group/cluster shocks can accelerate CRs. The subsequent $p p$ collisions between the shock-accelerated CRs and the thermal atomic nuclei in the gaseous environment is the major mechanism that generates HE neutrinos in these systems.

Here, we consider this scenario of both galactic scale shocks in the galactic ISM and group/cluster scale shocks in the intergalactic gas across redshifts. Whereas in a previous study (Kashiyama \& Mészáros 2014) only major galaxy mergers (mergers of two galaxies of approximately the same size) at $z \sim 1$ were considered, here take into account the redshift evolution of the halo merger rate, and consider both galaxy and cluster mergers, including both major and minor mergers (the latter being those where the participating galaxies or clusters have mass ratios $\zeta \neq 1$ ). We calculate the $\mathrm{CR}$ productions in the corresponding shocks at redshifts $0 \leq z \leq 10$ and we find that high redshift $(z \gtrsim 1-2)$ halo mergers contribute a significant part of the observed diffuse HE neutrinos and $\gamma$-rays. 


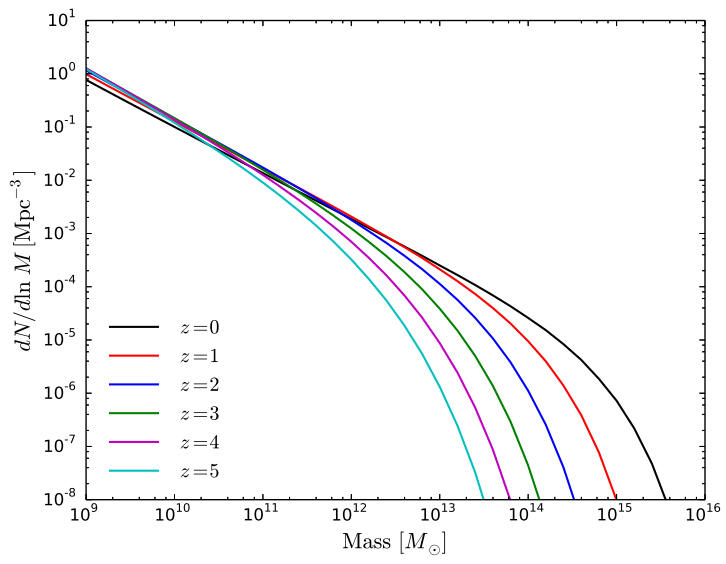

Figure 1. Dark matter halo mass function $d N / d \ln M$ at $z=0,1,2,3,4,5$. Here, we use the fitting formula from Sheth \& Tormen (1999).

This paper is organized as follows. In $\S 2$, we introduce the halo mass function and the halo distribution that is used in the following sections. The merger rate and the CR energy input rate are given in $\S 3$. In $\S 4$ we discuss the redshift dependence of the $\mathrm{CR}$ maximum energies and the neutrino product efficiency, and we calculate the resulting neutrino and the $\gamma$-ray fluxes. The results and implications are discussed in $\S 6$. Throughout, we assume a standard flat- $\Lambda$ CDM universe with present-day density parameter $\Omega_{m, 0}=0.3$ and Hubble parameter $H_{0}=71.9 \mathrm{~km} \mathrm{~s}^{-1} \mathrm{Mpc}^{-1}$ (Bonvin et al. 2017).

\section{HALO MASS FUNCTION}

Using the formalism of Press \& Schechter (1974), the halo mass function, the number of dark matter halos per unit comoving volume contained within the logarithmic mass interval $d \ln M$, is given by

$$
\frac{d N}{d \ln M}=\frac{\bar{\rho}}{M} f(\nu) \frac{d \ln \sigma_{M}^{-1}}{d \ln M},
$$

with the background matter density $\bar{\rho}=\Omega_{m, 0} \rho_{c r}$ where $\rho_{c r}=\left(3 H^{2} / 8 \pi G\right)$ is the critical density, and the variance $\sigma_{M}$ of the linear density contrast $\delta \equiv(\Delta \rho / \bar{\rho})$ is smoothed over the scale $R=\left(\frac{3 M}{4 \pi \bar{\rho}}\right)^{1 / 3}$ :

$$
\sigma_{M}^{2}=\int \frac{k^{2} d k}{2 \pi^{2}} P(k)|\hat{W}(k R)|^{2} .
$$

Here, $P(k)$ is the linear matter power spectrum we calculate following Lewis et al. (2000), and $\hat{W}(k R)=$ $3 j_{1}(k R) / k R$ for a top-hat filtering function. The significance $\nu=\delta_{c} / \sigma_{M}$ is related to the linear critical density $\delta_{c}$ above which virialized halos can form ${ }^{2}$. In the

${ }^{2}$ In some references, e.g. in Sheth \& Tormen (1999), $\nu=$ $\left(\delta_{c} / \sigma_{M}\right)^{2}$ is used instead of our definition here. spherical collapse model, for example, a spherical region of radius $R$ collapses and virializes at redshift $z$ when the smoothed linear overdensity $\delta_{R}(\boldsymbol{r}, z)$ exceeds $\delta_{c, 0} \approx 1.686$. In the flat $\Lambda$ CMD Universe, the linear growth factor, the time evolution of the linear density contrast, is given by

$$
\begin{aligned}
\mathcal{D}(z)=\frac{\delta_{c}(z)}{\delta_{c, 0}} & \propto \frac{5}{2} \Omega_{m, 0} \sqrt{\Omega_{m, 0}(1+z)^{3}+1-\Omega_{m, 0}} \\
& \times \int_{z}^{\infty} \frac{1+z^{\prime}}{\left[\Omega_{m, 0}\left(1+z^{\prime}\right)^{3}+1-\Omega_{m, 0}\right]^{3 / 2}} d z^{\prime} .
\end{aligned}
$$

We normalize $\mathcal{D}(z)$ to be unity at $z=0$.

For the multiplicity function $f(\nu)$, we adapt the Sheth-Tormen (Sheth \& Tormen 1999) fitting formula, expressed in the form

$$
f_{S-T}(\nu)=A \sqrt{\frac{2 a}{\pi}}\left[1+\left(\nu^{2} a\right)^{-p}\right] \nu \exp \left[-\frac{a \nu^{2}}{2}\right],
$$

with parameters $A=0.3222, a=0.707$, and $p=$ 0.3 that provide the the best fit to numerical $\mathrm{N}$ body simulations (Jenkins et al. 2001; Reed et al. 2006; Murray et al. 2013). We show the resulting mass function $\frac{d N}{d \ln M}$ for redshifts between $z=0$ and $z=5$ in figure 1. Our mass function slightly underestimates that from the N-body simulations at high masses of $\gtrsim 10^{15} M \odot$, but it does not affect the main results of this paper. We shall use the mass functions in the following sections to estimate the redshift evolutions of galactic radius, gas density and shock velocity.

\section{MERGER RATE AND COSMIC-RAY LUMINOSITY DENSITY}

In this section, we calculate the $\mathrm{CR}$ input rate due to galaxy and halo mergers by using the halo mass function we have obtained in $\S 2$, and we estimate the energy converted into CRs from shocks in the gas component of the merging halos as follows.

There are three time scales characterizing the CR acceleration due to galactic halo mergers: the age of the Universe $t_{\text {age }}$, the halo merger time $t_{\text {merger }}$ which corresponds to the average time required to undergo one merger, and the CR injection time (that is the shockcrossing time) $t_{\mathrm{dyn}}$, which are, for a merger that happens at redshift $z$, given by

$$
\begin{aligned}
t_{\text {age }} & =\int_{z}^{\infty}\left|\frac{d t}{d z^{\prime}}\right| d z^{\prime} \\
t_{\text {merger }} & =\left[\int d \zeta \frac{d N_{m}}{d z d \zeta}\left|\frac{d z}{d t}\right|\right]^{-1} \\
t_{\text {dyn }} & =\lambda \frac{R_{g}(z)}{v_{s}(z)} .
\end{aligned}
$$

Here, $|d t / d z|=1 /[(1+z) H(z)], d N_{m} / d z d \zeta$ (given by 
Fialkov \& Loeb 2016; Fakhouri et al. 2010) is the dimensionless merger rate per redshift interval $d z$ and per unit halo mass ratio $\zeta, R_{g}(z)$ is the mean galaxy radius, and $\lambda \sim 1$ parametrizes the orientation and geometrical uncertainty of the galaxy merger. With these time scales, the probability that a halo with mass $M$ experiences merger within the age of the universe is given by $P(M, z)=\exp \left(-t_{\text {merger }} / t_{\text {age }}\right)$. Hence, assuming that the CRs are mainly protons, the comoving $\mathrm{CR}$ energy input rate per the logarithm of the CR energy $\varepsilon_{p}$ is

$$
\begin{aligned}
\varepsilon_{p} Q_{\varepsilon_{p}}(z)=\frac{E_{\text {merger }}}{t_{\text {age }} \mathcal{C}}= & \epsilon_{p} \mathcal{C}^{-1} \int_{M_{\min }}^{M_{\max }} d M \\
& {\left[\frac{1}{2} \xi_{g}(M, z) M v_{s}^{2}\right] \frac{d N}{d M} \frac{P(M, z)}{t_{\text {age }}}, }
\end{aligned}
$$

where $\xi_{g}\left(M_{h}, z\right)=M_{\text {gas }} / M_{h}$ is the mass fraction in gas form, $\epsilon_{p}$ is the CR energy fraction (nominally taken as $0.1)$ and $\mathcal{C}=\ln \left(\varepsilon_{p}^{\max } / \varepsilon_{p}^{\min }\right)$ is the normalization factor for a standard flat CR spectrum $N\left(\varepsilon_{p}\right) \propto \varepsilon_{p}^{-2}$. For $z \sim$ 1, the typical maximum energy, $\varepsilon_{p}^{\max }$, is $\sim 10^{17} \mathrm{eV}$ and $\mathcal{C} \simeq 18.4$ (Kashiyama \& Mészáros 2014). However, $\varepsilon_{p}^{\max }$ varies with redshift, as we discuss in the next section.

\subsection{Gas-mass fraction $\xi_{g}(M, z)$}

The gas-mass fraction $\xi_{g}$ of dark matter halos depends on the star formation rate (SFR) and on the stellar mass $M_{*}=\chi_{*}\left(M_{h}, z\right) M_{h}$. Here, we obtain $\chi_{*}=M_{*} / M_{h}$ from the $M_{*}\left(M_{h}\right)$ function inferred from observations by Behroozi et al. (2013) ${ }^{3}$. We also use the gas fraction in normal galaxy $f_{g}=M_{\text {gas }} /\left(M_{\text {gas }}+M_{*}\right)^{4}$ measured in Sargent et al. (2014). Combining the two observational results, we have constructed the redshift evolution of the gas-mass fraction in dark matter halos. That is, the gas-mass fraction $\xi_{g}$ is related to $f_{g}$ through $\xi_{g}^{\text {evo }}=$ $M_{\text {gas }} / M_{h}=\frac{\chi_{*} f_{g}}{1-f_{g}}$, and using Eq. (26) in Sargent et al. (2014), we find that

$$
\xi_{g}^{\mathrm{evo}}=\chi_{*} \frac{f_{g}}{1-f_{g}}=\chi_{*} \frac{K}{M_{*}^{1-\beta^{\prime}}} \mathrm{SSFR}^{\beta^{\prime}}
$$

where $K=10^{\alpha_{\mathrm{SFR}}}$ is a constant and the quantity $\mathrm{SSFR}$ (specific star formation rate) is the star formation rate per unit galaxy stellar mass. For the gas fraction in normal galaxies we use the parameters $\left(\alpha_{\mathrm{SFR}}, \beta^{\prime}\right)=$ $(9.22 \pm 0.02,0.81 \pm 0.03)$, together with the expression for sSFR given in the appendix of Sargent et al. (2014).

\footnotetext{
${ }^{3}$ In Behroozi et al. (2013), the $M_{*}-M_{h}$ relation from $z=0-8$ is parameterized by equation (3). Here, we extend the domain of that function to $z=10$ considering that the uncertainty from high-redshift contributions is small.

${ }^{4}$ In Sargent et al. (2014), the gas fraction is written as $f_{\text {mol }}$ instead.
}

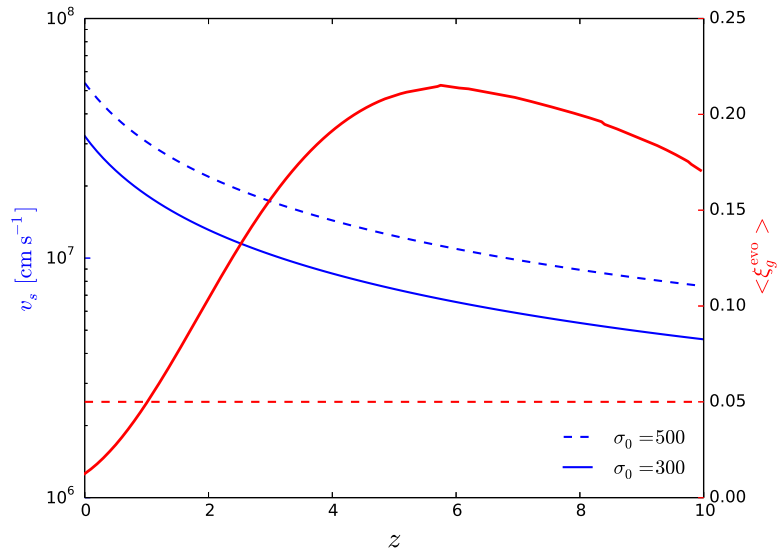

Figure 2. Redshift-dependent gas fraction $\xi_{g}(z)$ (red solid line) compared to a constant gas fraction $\xi_{g}=0.05$ (red dashed line) for redshift-dependent shock velocity with $\sigma_{0}=$ 300 (blue solid line) and with $\sigma_{0}=500$ (blue dashed line), respectively.

In Fig. 2 (see red curves), we show the redshift evolution of the mean gas-mass fraction,

$$
\left\langle\xi_{g}^{\mathrm{evo}}\right\rangle=\frac{\int \xi_{g}^{\mathrm{evo}} \frac{d N}{d M} d M}{\int \frac{d N}{d M} d M}
$$

as well as the constant gas fraction, $\xi_{g}=0.05$.

In our calculation, we take the lower and upper limit of the integration in Eq. (6) as $M_{\min }=10^{10} \mathrm{M}_{\odot}$ and $M_{\max }=10^{15} \mathrm{M}_{\odot}$, respectively. There are two main reasons to choose the lower bound $10^{10} \mathrm{M}_{\odot}$. First, considering the applicability of the $M_{*}\left(M_{h}\right)$ relation from Behroozi et al. (2013) and the gas fraction function (Eq. 7), it is safe to truncate the halo mass at $M_{h} \sim 10^{10} \mathrm{M}_{\odot}$. Typically, dwarf galaxies reside in halos with mass less than $10^{10} \mathrm{M}_{\odot}$ and we only have the constraints from observations at $z \simeq 0$. In our model, we consider the contribution from galaxy mergers up to the redshift $z=10$ where the $M_{*}\left(M_{h}\right)$ function is not well tested for the lower halo masses. Also, the gas fraction function (Eq. 7) is modeled from (normal) star-forming galaxies (Sargent et al. 2014) and may not be valid for dwarf galaxies. Second, we estimate the low-mass halo contribution to CR luminosity density by extending the lower bound to $10^{8} \mathrm{M}_{\odot}$ and found that the the contribution from $10^{8}-10^{10} \mathrm{M}_{\odot}$ halos is $\lesssim 10 \%$ of the total luminosity density in the low redshift $(z \lesssim 3)$, which implies that the conclusion of this paper does not depend sensitively on the mass range.

\subsection{Shock velocity $v_{s}$}

In the hierarchical clustering of large-scale structure scenario, the galactic-size halos are contained inside larger cluster-size halos. The peculiar velocities of the galactic-size halos are, therefore, of order of the virial velocity of the cluster-sized halo. Here, we approximate 
the shock velocity of the galaxy merger from the pairwise velocity dispersion projected along the line of approach of two galaxies. For galaxies with a luminosity $L \approx L^{*}$ (where $L^{*}$ is the characteristic luminosity), Davis \& Peebles (1983) showed that the two-point correlation function at $r<20 h^{-1} \mathrm{Mpc}$ can be approximated by a power law

$$
\xi(r)=\left(\frac{r}{r_{0}}\right)^{\gamma}
$$

where $\gamma \approx 1.7$ and $r_{0} \approx 5 h^{-1} \mathrm{Mpc}$ is the correlation length, inside which galaxies are strongly correlated. Combining the hierarchical form of the three-point correlation function of galaxies (Groth \& Peebles 1977) and the cosmic virial theorem derived from the Layzer-Irvine equation, the collision (or shock) velocity can be written as

$$
\begin{aligned}
v_{s} & =\sqrt{\bar{\sigma}^{2}(r)} \\
& \simeq \sigma_{0}\left(\frac{r_{0}}{5 h^{-1} \mathrm{Mpc}}\right)^{\gamma / 2}\left(\frac{r}{1 h^{-1} \mathrm{Mpc}}\right)^{-\gamma} \mathrm{km} \mathrm{s}^{-1}(.10)
\end{aligned}
$$

Given the average density of halos, we can estimate the average separation of galaxies through

$$
r=\left(\int \frac{d N}{d M} d M\right)^{-1 / 3} .
$$

For our calculation it is necessary to consider the redshift dependance of the correlation function $\xi(r)$ in the nonlinear regime. As a useful approximation, we adopt the stable clustering (SC) hypothesis (Peebles 1974; Davis \& Peebles 1977), in which only the size (or separation between structures) of the clusters changes in time while the internal density structure of clusters stays intact. This leads to $\xi(r, z) \propto(1+z)^{\gamma-3}$ and $r_{0} \propto$ $(1+z)^{-(3-\gamma) / \gamma}$. Note that we only need the evolution of the nonlinear scale $r_{0}$, which is defined by $\xi\left(r_{0}, z\right)=$ 1. A more accurate treatment (Hamilton et al. 1991) describes the evolution of $\xi(r, z)$ from the linear to the nonlinear regime, and this treatment was generalized by Peacock \& Dodds (1996) and by Smith et al. (2003) using another formula for the nonlinear function. This generalized method gives $\xi(r, z)$ in the quasilinear regime and confirms that $\xi(r, z) \propto(1+z)^{\gamma-3}$ is valid in the nonlinear limit as well. Therefore, in this paper, we use $r_{0}^{\mathrm{SC}} \propto(1+z)^{-(3-\gamma) / \gamma}$ in Eq. (10) to find the shock velocities. We show the redshift dependence of the shock velocity $v_{s}(z)$ in Fig. 2 (see blue curves).

Note that, in our approximation of the shock velocity [Eq. (10)], the galaxy separation $r$ given by Eq. (11) is overestimated, since this latter equation takes an average of the galaxies in a cosmological volume including clusters and voids. The mean separation of the galaxies in clusters, therefore, must be smaller than the value that we have adopted here, and this overestimate of $r$ would give a slight underestimate of $v_{s}$ inside clusters. As a possible way to correct for this, we note that redshift surveys give $\sigma_{0} \sim 500$ (Jing et al. 1998; Hawkins et al. 2003; Zehavi et al. 2002) as an average value for mergers in clusters. A qualitatively appropriate correction for the cluster shock velocity may be obtained by scaling up $\sigma_{0}$ in Eq. (10) from the local average value, concluding that a realistic value of $\sigma_{0}$ for clusters is in the range $500 \lesssim \sigma_{0} \lesssim 600$. In terms of rates, most galaxy mergers occur in the smaller mass halos containing fewer galaxies, as opposed to large clusters. Considering the observational and theoretical uncertainties, we expect that the values of $\sigma_{0}$ lie in the range of $100 \lesssim \sigma_{0} \lesssim 1000$, and we take $300 \lesssim \sigma_{0} \lesssim 500$ as fiducial values.

\section{NEUTRINO AND $\gamma$-RAY PRODUCTION}

Since in our model we need to consider the neutrino $/ \gamma$ ray production rate up to redshift $z=10$, we introduce here the redshift evolution function of the gas density, $g(z)=n(z) / n(z=0)$. To define this function we use the result that a sphere of gas will collapse and virialize once its density exceeds the value $1.686 \mathcal{D}(z)^{-1} \rho_{c}(z)$ (Peebles 1980). The mean density of the virialized gas is $\Delta_{c} \rho_{c}(z)$, where $\rho_{c}(z)=3 H(z)^{2} /(8 \pi G)$, and an approximation of $\Delta_{c}(z)$ is $\Delta_{c} \approx 178 \Omega_{m}^{0.45}$ (Eke et al. 1998), where $\Omega_{m}=\Omega_{m, 0}(1+z)^{3} /\left[\Omega_{m, 0}(1+z)^{3}+1-\Omega_{m, 0}\right]$. Since clusters are the largest virialized objects in the universe, we take $\rho_{\mathrm{cl}}(z)=g(z) n_{\mathrm{cl}, 0} m_{p} \propto \Delta_{c} \rho_{c}(z)$, and we assume that galaxies, halos and clusters all share a universal $g(z)$,

$g(z)=\frac{\Delta_{c} \rho_{c}(z)}{\Delta_{c, 0} \rho_{c}(0)}=(1+z)^{1.35}\left[\Omega_{m, 0}(1+z)^{3}+1-\Omega_{m, 0}\right]^{0.55}$.

In the following sections, the relations $n_{\mathrm{g}}(z)=$ $g(z) n_{\mathrm{g}, 0}, n_{\mathrm{cl}}(z)=g(z) n_{\mathrm{cl}, 0}$ will be used for the postshock magnetic field and $p p$ optical depth.

\subsection{Galaxy mergers}

The maximum energy of CRs accelerated in the merger shocks will also evolve with $z$ due to the redshift dependance of the typical galactic radius and magnetic field characterizing the shocks. The magnetic field behind the shock in a galaxy merger is commonly parametrized as a fraction of the ram pressure (Kashiyama \& Mészáros 2014), $B_{s}^{2} / 8 \pi=\frac{1}{2} \epsilon_{B} n_{\mathrm{g}} m_{p} v_{s}^{2} \propto$ $\rho v_{s}^{2}$. This implies a magnetic field

$$
\begin{aligned}
B_{s} & =\sqrt{4 \pi \epsilon_{B} n_{\mathrm{g}, 0} m_{p} g(z) v_{s}^{2}} \\
& \simeq 14 \epsilon_{B,-2}^{1 / 2} n_{\mathrm{g}, 0}^{1 / 2} g(z)^{1 / 2} \times\left(\frac{v_{s}}{300 \mathrm{~km} \mathrm{~s}^{-1}}\right) \mu \mathrm{G} .
\end{aligned}
$$

The magnetic field in the disk region is expected to be higher than that in the halo region. Although details 
depend on the geometry, for simplicity, we assume that a reasonably strong magnetic field is expected over scales between the galaxy radius $R_{\mathrm{g}}$ and a gas scale height $h_{\mathrm{g}}$, which is taken as the characteristic scale height $h \sim$ $\left(3 h_{\mathrm{g}} R_{g}^{2} / 2\right)^{1 / 3}$ in this work. Then, the maximum CR energy is estimated to be (Drury 1983)

$$
\begin{gathered}
\varepsilon_{p}^{\max } \sim \frac{3}{20} e B_{s} h \frac{v_{s}}{c} \simeq 1.3 \times 10^{16} \mathrm{eV}\left(\frac{B_{s}}{30 \mu G}\right) \times \\
\left(\frac{h}{3 \mathrm{kpc}}\right)\left(\frac{v_{s}}{300 \mathrm{~km} \mathrm{~s}^{-1}}\right) .
\end{gathered}
$$

The CRs are advected to the far downstream, and produce neutrinos and $\gamma$-rays during the advection. In reality, one needs to calculate neutrinos and $\gamma$-rays from the post-shock region especially when the $p p$ optical depth in the $\mathrm{CR}$ acceleration region is dominant. The emissions occur during $t_{\mathrm{dyn}} \sim h / v_{s} \simeq$ $9.8 \mathrm{Myr}(h / 3 \mathrm{kpc})\left(300 \mathrm{~km} \mathrm{~s}^{-1} / v_{s}\right)$. In this work, for simplicity, we take the CR reservoir limit, in which the CRs mostly escape into the ISM and the neutrino and $\gamma$-ray production mainly occurs in the ISM.

After the CRs are accelerated in the shock, they will propagate in the host galaxy and cluster. In this process, neutrinos and $\gamma$-rays are generated from pions produced in inelastic $p p$ collisions. The meson production efficiency is $1-\exp \left(-f_{p p}\right)$ where $f_{p p}=c \kappa_{p p} \sigma_{p p} g(z) \sum n_{i, 0} t_{i}$ is the effective $p p$ optical depth. In this expression, $n_{i, 0}$ is the local gas density of the medium, e.g. galaxies and clusters, $\sigma_{p p}=\sigma_{p p}\left(\varepsilon_{p}\right)$ is the $p p$ cross section given by Kafexhiu et al. (2014), $\kappa_{p p}=0.5$ is the inelasticity coefficient and $g(z)$ (see Eq. (12)) represents the redshift evolution of the gas density.

Let us consider galaxies that are merging at $z$. Inside the merged galaxy, $f_{p p}^{\mathrm{g}}$ is determined by the time spent by the CRs undergoing $p p$ collisions, which depends on the CR injection time and and the diffusion time in the medium. The dynamical time is given by the third of Eq. (5), while the diffusion time is $t_{\text {diff }}=h(z)^{2} /\left(6 D_{\mathrm{g}}\right)$, where $h(z)$ is the effective gas size at $z$ and $D_{g}$ is the diffusion coefficient in the galactic ISM gas. Here, we use a combined large and small angle diffusion expression as in Senno et al. (2015), $D=D_{c}\left[\left(\varepsilon / \varepsilon_{c, g}\right)^{1 / 2}+\left(\varepsilon / \varepsilon_{c, g}\right)^{2}\right]$, where $D_{c}=\operatorname{cr}_{L}\left(\varepsilon_{c, g}\right) / 4$ and $\varepsilon_{c, g}$ is determined from $r_{L}\left(\varepsilon_{c, g}\right)=l_{c} / 5$. Here, $r_{L}$ and $l_{c}$ are the Larmor radius and coherence length in the galaxy environment respectively. For local normal galaxies, the gas density in the disk is $n_{\mathrm{g}, 0} \sim 1 \mathrm{~cm}^{-3}$, whereas the average density in the galactic halo is smaller, $n_{\mathrm{g}, 0} \sim 0.1 \mathrm{~cm}^{-3}$. The magnetic field of local normal galaxies is $\sim 4 \mu G$ and that of starforming galaxies is $\sim 6 \mu G$, respectively (Keeney et al. 2006; Crocker 2012). For the density and magnetic field of merging galaxies, we take values higher than those of normal galaxies, since the galaxies may enter the starburst phase during the merger. Specifically we adopt a mean value $n_{\mathrm{g}, 0}=1 \mathrm{~cm}^{-3}$. Thus, we have

$$
t_{\mathrm{diff}} \simeq 3.2 \times 10^{5} \mathrm{yr}\left(\frac{h(z)}{3 \mathrm{kpc}}\right)\left[\left(\varepsilon / \varepsilon_{c, g}\right)^{1 / 2}+\left(\varepsilon / \varepsilon_{c, g}\right)^{2}\right]^{-1}
$$

where

$$
\varepsilon_{c, g} \simeq 1.7 \times 10^{9} \mathrm{GeV}\left(\frac{h(z)}{3 \mathrm{kpc}}\right)\left(\frac{B_{g}}{30 \mu G}\right) .
$$

Calculations of the neutrino and $\gamma$-ray emission depend on details of the spatial extension and time evolution of the shock region and its surrounding environment. The latter is also modified by the shock, star-formation, and outflow. For simplicity, we treat a double-galaxy merger system as one CR reservoir for the injection by the merger shock, which is conservative since there should also be the emissions from the accelerator. A similar treatment for neutrino sources with active accelerators is used in the galaxy cluster model (Murase et al. 2008; Fang \& Murase 2018). Then, the effective $p p$ optical depth is estimated to be $f_{p p}^{\mathrm{g}}=\kappa_{p p} c g(z) n_{\mathrm{g}, 0} \sigma_{p p} \min \left[t_{\mathrm{dyn}}, t_{\mathrm{diff}}\right] \simeq$ $0.24 g(z)\left(\frac{n_{\mathrm{g}, 0}}{1 \mathrm{~cm}-3}\right)\left(\frac{\sigma_{p p}}{50 \mathrm{mb}}\right)\left(\frac{\min \left[t_{\mathrm{dyn}}, t_{\mathrm{diff}}\right]}{10 \mathrm{Myr}}\right)$ in the merging galaxy system. The ambient magnetic field energy may be taken to be a fraction of the merging galaxy system's virial energy, as $B_{\mathrm{g}}^{2} R_{\mathrm{g}}^{3} \propto G M_{\mathrm{g}}^{2} / R_{\mathrm{g}}$, i.e. $B_{\mathrm{g}} \propto \rho_{\mathrm{g}} R_{\mathrm{g}} \propto g(z) R_{\mathrm{g}}(z)$.

The typical galactic radii evolve with redshift $z$, and considering the merger history of galaxies, it is apparent that the mean radii of galaxies at $z$ should be smaller than $R_{\mathrm{g}, 0} /(1+z)$, where $R_{\mathrm{g}, 0} \approx 10 \mathrm{kpc}$ is the radius of local Milky Way-like galaxies. Shibuya et al. (2015) studied the redshift evolution of the galaxy effective radius $r_{e}$ using Hubble Space Telescope (HST) samples of galaxies at $z=0-10$, finding $r_{e} \propto(1+z)^{-1.0}-(1+z)^{-1.3}$ with $r_{e} \propto(1+z)^{-1.10 \pm 0.06}$ as a median. Hence, in this paper, we assume that the average galaxy radius evolves with respect to $z$ as $R_{\mathrm{g}}=R_{\mathrm{g}, 0}(1+z)^{-1.10}$.

As for the scale height $h_{g}(z)$, based on the surface photometry analysis of edge-on spiral galaxies, e.g. NGC 4565 , it has been shown that the scale height of gas in local disk galaxies is approximately $h_{g, 0} \approx 300-400$ pc (Bahcall \& Soneira 1980; Van Der Kruit \& Searle 1981). Later studies of NGC 891 (Kylafis \& Bahcall 1987), NGC 5097(Barnaby \& Thronson Jr 1992) etc. also agree with this estimate. Considering that a merger can lead to entering a star-forming phase, we assume $h_{g, 0}=500 p c$ and we assume the same redshift dependence as for $R_{\mathrm{g}}$, e.g. $h_{\mathrm{g}}(z)=(1+z)^{-1.10} h_{\mathrm{g}, 0}$. Then we take $h=\left(3 h_{\mathrm{g}} R_{\mathrm{g}}^{2} / 2\right)^{1 / 3}$.

\subsection{Interactions in the host cluster and cluster mergers}


After escaping the galaxy, the CRs may continue to collide with the gas of the host cluster, where $t_{\text {diff }}=R_{\mathrm{cl}}(z)^{2} /\left(6 D_{\mathrm{cl}}\right)$. Here, we assume a magnetic field $B_{\mathrm{cl}, 0} \approx 1 \mu G$ with a coherence length $l_{c, \mathrm{cl}} \approx 30 \mathrm{kpc}$. This implies $\varepsilon_{c, \mathrm{cl}} \approx 5.6 \times 10^{9} \mathrm{GeV}$. For a cluster of mass $10^{15} \mathrm{M}_{\odot}$, the virial radius is $R_{\mathrm{cl}, 0}=\left(3 M /\left(4 \pi \rho_{\mathrm{cl}, 0}\right)\right)^{1 / 3} \approx 2.1 \mathrm{Mpc}$. Since $R_{\mathrm{cl}}$ is the approximate boundary of clustered/correlated galaxies, it should have the same redshift dependence as $r_{0}^{\mathrm{SC}}$. Using the stable clustering approximation, we obtain $R_{\mathrm{cl}} \propto(1+z)^{-(3-\gamma) / \gamma}$. Similarly, we can calculate the diffusion time in clusters as $t_{\text {diff,cl }}=1.2\left[\left(\varepsilon / \varepsilon_{c, \mathrm{cl}}\right)^{1 / 2}+\left(\varepsilon / \varepsilon_{c, \mathrm{cl}}\right)^{2}\right]^{-1}$ Gyr. Assuming that the injection time of CRs $\left(t_{\mathrm{inj}}\right)$ at redshift $z$ is the cluster age (of order the Hubble time) $t_{\text {age }}(z)$, likewise we obtain the optical $\operatorname{depth} f_{p p}^{\mathrm{cl}}=\kappa_{p p} c g(z) n_{\mathrm{cl}, 0} \sigma_{p p} \min \left[t_{\mathrm{inj}}, t_{\mathrm{diff}, \mathrm{cl}}\right] \simeq$ $0.24 g(z)\left(\frac{n_{\mathrm{cl}, 0}}{10^{-3} \mathrm{~cm}^{-3}}\right)\left(\frac{\sigma_{p p}}{50 \mathrm{mb}}\right)\left(\frac{\min \left[t_{\mathrm{age}}, t_{\mathrm{diff}}\right]}{10 \mathrm{Gyr}}\right)$, where $n_{\mathrm{cl}, 0}$, the intercluster gas density, is assumed to have the typical value $n_{\mathrm{cl}, 0} \sim 10^{-4}-10^{-2} \mathrm{~cm}^{-3}$ (Croston et al. 2008), which can be higher in cooling core clusters. The magnetic field may also depend on $z$ as $B_{\mathrm{cl}} \propto \rho_{\mathrm{cl}} R_{\mathrm{cl}} \propto g(z) R_{\mathrm{cl}}(z)$.

Halo mergers will also lead to galaxy group and galaxy cluster mergers, after some halos have grown above a certain size which may be taken to be roughly of order $M_{h} \sim 10^{13} \mathrm{M}_{\odot}$. We simplify the calculations as follows. For low-mass mergers, we expect that the $p p$ interactions occur mainly in gas with an ISM density characteristic of galaxies, while for high-mass mergers the $p p$ interactions occur mainly in gas with an IGM density characterizing the intra-cluster gas. In addition, there will be a component of $p p$ interactions due to low-mass merger CRs which escape from the colliding galaxy system into the IGM. Thus, we expect that the all-flavor neutrino production rate consists of a galaxy part $\varepsilon_{\nu} Q_{\varepsilon_{\nu}}^{(\mathrm{g})}$ and a cluster/group part $\varepsilon_{\nu} Q_{\varepsilon_{\nu}}^{(\mathrm{cl})}$ plus a weaker galaxy-cluster term,

$$
\begin{aligned}
\varepsilon_{\nu} Q_{\varepsilon_{\nu}}^{(\mathrm{g})}= & \frac{1}{2}\left(1-e^{-f_{p p}^{\mathrm{g}}}\right) \varepsilon_{p} Q_{\varepsilon_{p}}^{(\mathrm{LM})} \\
\varepsilon_{\nu} Q_{\varepsilon_{\nu}}^{(\mathrm{cl})}= & \frac{1}{2}\left[\left(1-e^{-f_{p p}^{\mathrm{cl}}}\right) \varepsilon_{p} Q_{\varepsilon_{p}}^{(\mathrm{HM})}\right. \\
& \left.+\eta\left(1-e^{-f_{p p}^{\mathrm{cl}}}\right) e^{-f_{p p}^{\mathrm{g}}} \varepsilon_{p} Q_{\varepsilon_{p}}^{(\mathrm{LM})}\right],
\end{aligned}
$$

where the energies of the neutrinos and CR protons are related by $\varepsilon_{\nu} \approx 0.05 \varepsilon_{p}$. Note that the luminosity density evolution of neutrinos and $\gamma$-rays is different from that of CRs in general. In the first line of Eq. $17 \varepsilon_{p} Q_{\varepsilon_{p}}^{(\mathrm{LM})}$ is the CR input rate (see Eq. 6) from galaxy mergers in lowmass (LM) halos, e.g. $\left[10^{10} \mathrm{M}_{\odot}, 10^{13} \mathrm{M}_{\odot}\right]$. In the second line $\varepsilon_{p} Q_{\varepsilon_{p}}^{(\mathrm{HM})}$ is the $\mathrm{CR}$ input rate of the high-mass (HM) halo mergers, in the interval $\left[10^{13} \mathrm{M}_{\odot}, 10^{15} \mathrm{M}_{\odot}\right]$. The factors $\frac{1}{2}\left(1-e^{-f_{p p}^{\mathrm{i}}}\right) \varepsilon_{p} Q_{\varepsilon_{p}}^{(\mathrm{j})}$ are the neutrino luminosity density from CRs originating from mergers of mass $(j)$ in gas of density $i$. For our fiducial parameters, these two components constitute the largest fraction of the neutrino budget. Nevertheless, for completeness, we have included in the third line of Eq. 17 the sub-dominant effect due the CRs produced in galaxy mergers which may escape the host galaxies and collide with intra-cluster gas to produce neutrinos. (This can be important only if the $p p$ interactions in galaxy mergers are inefficient.) We introduce a parameter $\eta$ to represent the fraction of galaxy mergers that occur inside clusters which lead to some CRs escaping into the gas halo. This can occur preferentially at lower redshifts. Since the boundary between LM and HM is ambiguous and the fraction $\eta$ can change with redshift, this parameter is very uncertain, and may conservatively be estimated as between 0.1 and at most 0.5 . Fortunately, the contribution of this higher-order third component depending on $\eta$ is small compared to the first two components in Eq. 17, due to the factor $e^{-f_{p p}^{\mathrm{g}}}$. At $z=1$, the ratio between the third line and the first line is $\leq 10 \%$ even if $\eta$ is assumed to be unity, and it is increasingly negligible at higher redshift since $f_{p p}^{g}$ increases as the gas density increases. Therefore, the exact value of $\eta$ does not significantly influence our final results.

\section{DIFFUSE NEUTRINO AND $\gamma$-RAY SPECTRA}

With the above, we are able to determine the $\mathrm{CR}$ energy input rate, $\varepsilon_{\nu} Q_{\varepsilon_{\nu}}^{(\mathrm{g})}$ and $\varepsilon_{\nu} Q_{\varepsilon_{\nu}}^{(\mathrm{cl})}$. Figure 3 shows the $\mathrm{CR}$ input power over the whole mass interval $10^{10} \mathrm{M}_{\odot}-$ $10^{15} \mathrm{M}_{\odot}$ as a function of $z$ as well as the LM and HM components of $\left(\sigma_{0}=300, \xi_{g}^{\text {evo }}\right)$ scenario. As can be seen from the redshift distribution of the CR energy input, using a redshift evolving gas fraction $\xi_{g}^{\text {evo }}$, a significant fraction of this occurs at redshifts $z \gtrsim 3$, above which a significant $\gamma \gamma$ attenuation of the accompanying highenergy $\gamma$ rays at $\gtrsim 20-30 \mathrm{GeV}$ energies can be expected (e.g., Chang et al. 2016; Xiao et al. 2016). In addition, from Figure 3, we find that the high-mass and low-mass components are comparable in local mergers, implying that the cluster/group merger contribution is also important. Also, the galaxy merger contribution to the CR luminosity density is more important at $z \gtrsim 2$.

Given the neutrino input rate, the all-flavor neutrino flux can be expressed as (Murase et al. 2016)

$$
\varepsilon_{\nu}^{2} \Phi_{\varepsilon_{\nu}}=\frac{c}{4 \pi} \int \frac{\varepsilon_{\nu} Q_{\varepsilon_{\nu}}^{(\mathrm{g})}+\varepsilon_{\nu} Q_{\varepsilon_{\nu}}^{(\mathrm{cl})}}{(1+z)}\left|\frac{d t}{d z}\right| d z
$$

Based on the branching ratio between charged and neutral pions, the initial diffuse $\gamma$-ray energy spectrum is expected to be given by $\varepsilon_{\gamma}^{2} \Phi_{\varepsilon_{\gamma}}=\left.\frac{2}{3} \varepsilon_{\nu}^{2} \Phi_{\varepsilon_{\nu}}\right|_{\varepsilon_{\nu}=0.5 \varepsilon_{\gamma}}$. Since however the high-energy $\gamma$ rays can annihilate with lower energy photons, such as those from the extragalactic background light (EBL) and the cosmic microwave 


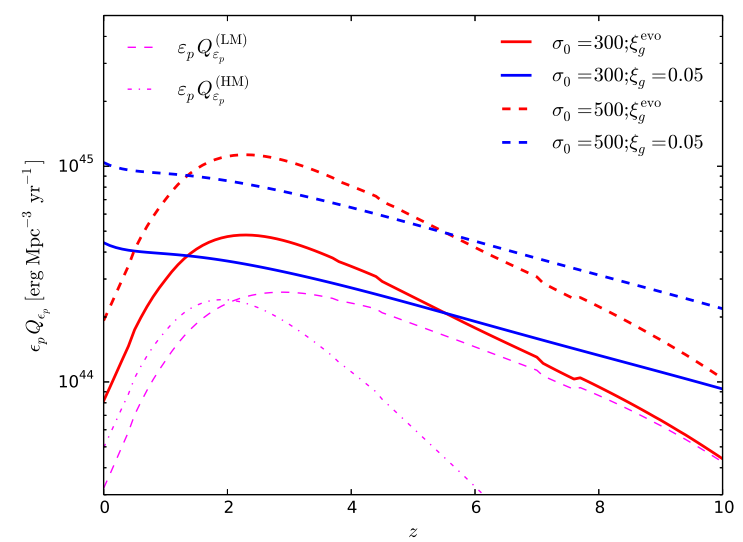

Figure 3. CR energy input rate versus redshift. The red lines correspond to a redshift-dependent gas fraction $\xi_{g}^{\text {evo }}$ and the blue lines are for a redshift-independent gas fraction $\xi_{g}=0.05$, while the solid lines are for $\sigma_{0}=300$ and the dashed are for $\sigma_{0}=500$, repectively. The dashed and dash-dotted magenta lines are LM and HM components of $\left(\sigma_{0}=300, \xi_{g}^{\text {evo }}\right)$ scenario. Here LM and HM denote the low-mass $\left(10^{10} \mathrm{M}_{\odot}-10^{13} \mathrm{M}_{\odot}\right)$ and high-mass $\left(10^{13} \mathrm{M}_{\odot}-10^{15} \mathrm{M}_{\odot}\right)$ intervals, respectively.

background (CMB), we introduce an attenuation factor $\exp \left[-\tau_{\gamma \gamma}\left(\varepsilon_{\gamma}, z\right)\right]$ to the integration, where $\tau_{\gamma \gamma}$ is the $\gamma \gamma$ optical depth at redshift $z$. In this paper, we use the optical depth provided by (Finke et al. 2010; Inoue et al. 2013) for low-redshift $(z \leq 5)$ and high-redshift $(z>5)$ inputs, respectively. The attenuated $\gamma$-ray flux is then

$$
\begin{aligned}
\varepsilon_{\gamma}^{2} \Phi_{\varepsilon_{\gamma}}=\frac{c}{4 \pi} \int \frac{2}{3} & {\left[\frac{\varepsilon_{\nu} Q_{\varepsilon_{\nu}}^{(\mathrm{g})}+\varepsilon_{\nu} Q_{\varepsilon_{\nu}}^{(\mathrm{cl})}}{(1+z)}\left|\frac{d t}{d z}\right|\right] } \\
& \times \exp \left[-\tau_{\gamma \gamma}\left(\varepsilon_{\gamma}, z\right)\right] d z
\end{aligned}
$$

with $\varepsilon_{p}=10 \varepsilon_{\gamma}(1+z)$. In addition, the electronpositron pairs produced in the $\gamma \gamma$ annihilations will subsequently scatter off the ambient diffuse photon backgrounds, leading to an electromagnetic cascade which in part compensates for the attenuation, while reprocessing the photon energy towards lower energies, which can be detected by, e.g. the Fermi-LAT instrument. In this paper, for simplicity, we use the universal form for the resulting cascaded $\gamma$-ray spectrum given by Berezinsky \& Smirnov (1975) (see also, e.g., Murase \& Beacom 2012; Senno et al. 2015),

$$
\varepsilon_{\gamma} \frac{d N}{d \varepsilon_{\gamma}} \propto G\left(\varepsilon_{\gamma}\right)= \begin{cases}\left(\frac{\varepsilon_{\gamma}}{\varepsilon_{\gamma}^{\text {br }}}\right)^{-1 / 2} & \varepsilon_{\gamma} \leq \varepsilon_{\gamma}^{\mathrm{br}} \\ \left(\frac{\varepsilon_{\gamma}}{\varepsilon_{\gamma}^{\text {cut }}}\right)^{-1} & \varepsilon_{\gamma}^{\mathrm{br}}<\varepsilon_{\gamma}<\varepsilon_{\gamma}^{\text {cut }}\end{cases}
$$

where $\varepsilon_{\gamma}^{\text {cut }}$ is defined by $\tau_{\gamma}\left(\varepsilon_{\gamma}^{\text {cut }}, z\right)=1$ and $\varepsilon_{\gamma}^{\text {br }}=$ $0.0085 \mathrm{GeV}(1+z)^{2}\left(\frac{\varepsilon_{\gamma}^{\text {cut }}}{100 \mathrm{GeV}}\right)^{2}$.

The all-flavor diffuse neutrino and $\gamma$-ray fluxes are plotted in Fig. 4, together with the IceCube observed astrophysical neutrinos. The red points and cyan points correspond to the all-flavor averaged neutrino flux (Aartsen et al. 2015, 2016) and the 6-year high energy starting-events (HESE) (Aartsen et al. 2017), respectively. The Fermi-LAT observed total extragalactic $\gamma$-ray background (EGB) (Ackermann et al. 2015) is shown by the blue points. The yellow area is the best-fit to the up-coming muon neutrinos scaled to three-flavor. Figure 4 shows the results for an assumed redshiftdependent gas fraction $\xi_{g}^{\mathrm{evo}}$, as illustrated in Fig. 4(a) for $\sigma_{0}=300$ and in Fig. 4(b) for $\sigma_{0}=500$, showing the effect of the corresponding different shock velocities $v_{s}$. In each figure, the magenta line represents the neutrino flux while the green line illustrates the corresponding $\gamma$-ray flux after cascading down. The galaxy and cluster contributions to the neutrino flux are plotted in dashed lines and dash-dotted lines. The non-blazar (Ackermann et al. 2016) component of the unresolved extragalactic gamma-ray background is shown as the pink area.

For illustration purposes, we consider next the corresponding results using the redshift-independent gas fraction $\xi_{g}=0.05$, which are shown in figures 5 (a) and 5 (b). The comparison between the galaxy and cluster components indicates that the high-energy neutrinos are dominantly produced by the propagation of CRs in the clusters. This is a consequence of the rapid redshift evolution of the galaxy radius, since the size of the host galaxy limits the maximum CR energy as well as the neutrino production efficiency by restricting the diffusion time. In addition, a redshift-dependent $\xi_{g}^{\text {evo }}$ boosts the $\mathrm{CR}$ budget to a relatively higher redshift $(z \approx 3)$, as can be seen from the red line in Fig.3, which as was expected leads to a reduction in the $\gamma$-ray flux. From these figures, we can also see that even with the moderate sensitivity of the results to the parameters $r_{0}$ and $\sigma_{0}$, the results can broadly fit a significant fraction of the IceCube data without violating the non-blazar EGB. Conversely, the $\gamma$-ray and neutrino fluxes are significantly constrained in this scenario, indicating that the halo and galaxy mergers can be regarded as promising sources of neutrinos in the context of multi-messenger studies.

\section{DISCUSSION}

In this work, we investigated the contribution of halo mergers to the diffuse neutrino and $\gamma$-ray backgrounds, and tested whether the non-blazar diffuse $\gamma$-ray background Fermi constraint is violated. Our results differ from previous work by Kashiyama \& Mészáros (2014) in that we studied both galaxy and cluster/group mergers out to higher redshifts, up to $z \approx 10$, by considering the redshift evolution of the average galactic radius, the shock velocity and the gas content inside the halos, as well as the galactic/intergalactic magnetic fields.

The redshift evolution of galaxy radius implies that 

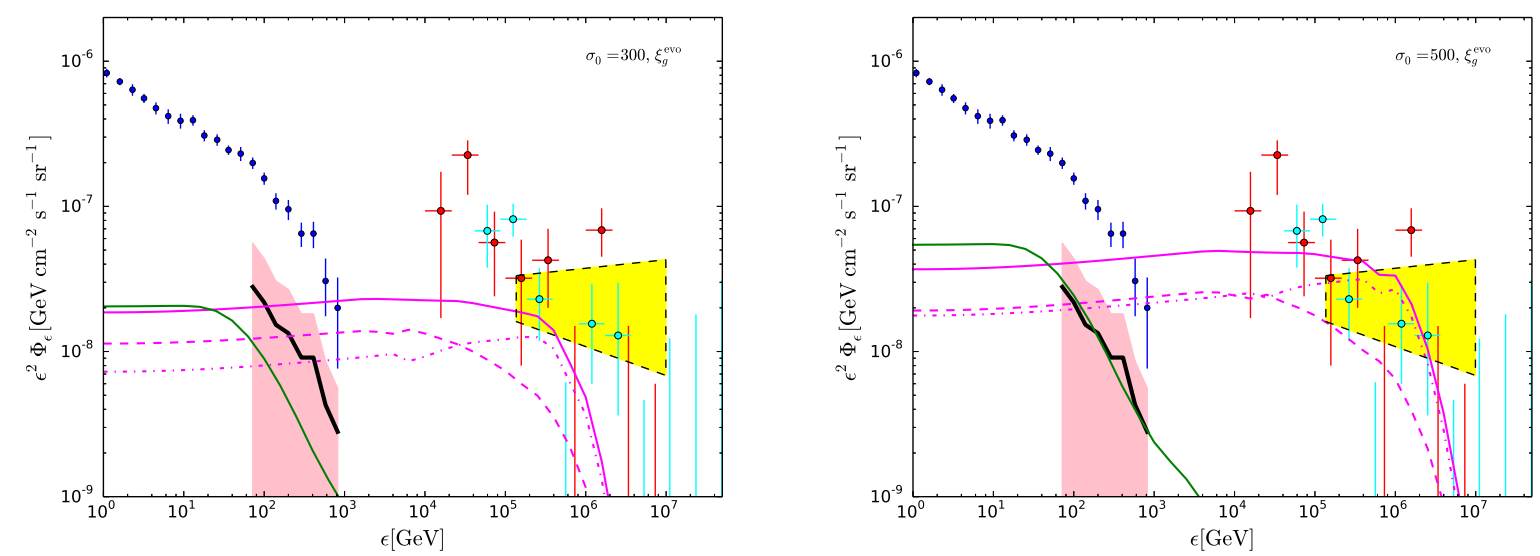

Figure 4. Left panel: Neutrino (all flavor) and $\gamma$-ray fluxes from halo mergers with redshift-evolving gas fraction $\xi_{g}^{\text {evo }}, R_{g, 0}=$ $10 \mathrm{kpc}, H_{g, 0}=500 \mathrm{pc}$. The shock velocity is obtained using $r_{0}^{\mathrm{sc}}(z)$ and $\sigma_{0}=300$. The magenta line is the neutrino spectrum while the green line is the corresponding $\gamma$-ray spectrum. Galaxy and cluster contributions to the neutrino flux are illustrated as the dashed and dash-dotted lines, respectively. Right panel: same as left panel except $\sigma_{0}=500$ is utilized for $v_{s}$.
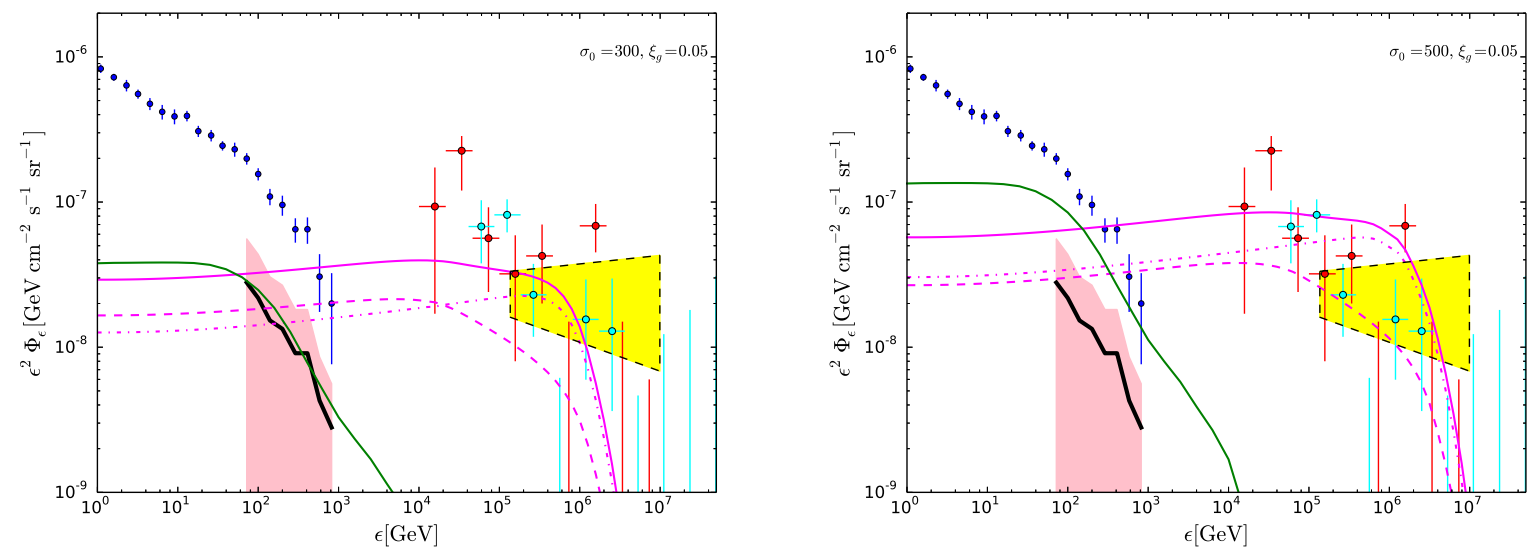

Figure 5. Left panel: same as Fig. 4 (a), $\sigma_{0}=300$, except that $\xi_{g}=0.05$ is used to estimate the redshift evolution of the halo gas fraction. Right panel: same as left figure except with $\sigma_{0}=500$.

there exist more protogalaxies, or equivalently more mergers at higher redshift. In fact, the merger rate calculated using our approximate approach verifies this conjecture, as well as being consistent with the Illustris simulations (Rodriguez-Gomez et al. 2015). Also, our estimates of the gas fraction $\xi_{g}^{\text {evo }}$ based on the correlation between the galactic gas content and the star formation rate shows that the gas in high-redshift halos is relatively denser than in the current epoch halos. The net effect is that high-redshift halo mergers can contribute a significant fraction of the cosmic rays that are capable of producing high-energy neutrinos, as shown in Fig. 3. This is crucial since the accompanying $\gamma$-ray photons in the ensuing $p p$ collisions at high redshifts can be sufficiently absorbed via $\gamma \gamma$ annihilations against CMB and EBL photons. In both cases with $\xi_{g}^{\text {evo }}$, our results indicate that high-redshift galaxy/halo mergers can explain a large fraction of the IceCube observed diffuse neutrinos up to $\sim \mathrm{PeV}$, with an accompanying $\gamma$ ray diffuse observed flux which is below the non-blazar Fermi constraints.

We note that according to our calculation, the diffuse flux of CRs that survive from energy losses via $p p$ collisions is less than $10^{-8} \mathrm{GeV} \mathrm{cm}{ }^{-2} \mathrm{sr}^{-1} \mathrm{~s}^{-1}$, which is lower than the observed CR flux around the knee or sub-ankle energy.

The CR acceleration efficiency $\epsilon_{p}$ is expected to be $\sim 0.1$ based on the diffusive shock acceleration theory. The redshift dependences of gas fraction $\xi_{g}^{\text {evo }}$ and galaxy radius are relatively well-modeled from current theories and observations, so our scenario can put a tighter constraint on the shock velocity of galaxy mergers. However, there are large uncertainties in the model. For example, the maximum energy depends on the magnetic field strength that is highly uncertain. On the other hand, the fiducial value of $\sim 10 \mathrm{PeV}$ is not far 
from the knee energy at $\sim 3 \mathrm{PeV}$, so our assumption is reasonable. One of the most important uncertainties is caused by the shock velocity. Our fiducial parameters $\left(\sigma_{0}=300\right.$ with $\left.\xi_{g}^{\text {evo }}\right)$ imply a lower neutrino flux compared to the observations. This could be overcome by assuming a higher velocity with a stronger magnetic field. Or it may be possible to achieve the IceCube flux at $\gtrsim 0.1 \mathrm{PeV}$ without exceeding the Fermi constraint by increasing the cluster contribution. However, the cluster contribution is more uncertain. Non-thermal emissions from merging/accreting clusters have been studied by various authors (e.g., Berrington \& Dermer 2003; Fujita \& Sarazin 2001). The Mach number of shocks on the cluster scales is so low due to the high temperature of the intra-cluster medium that the shock may not be strong enough to have a hard spectrum of $s \sim 2$.

We note that, in addition to mergers, also cluster accretion shocks and powerful jets from radio-loud AGNs can contribute to CR acceleration inside the clusters/groups, as considered in the previous literature (e.g., Murase et al. 2013; Fang \& Murase 2018, and references therein). One of the generic features of the $\mathrm{CR}$ reservoir scenario is that different possibilities for $\mathrm{CR}$ acceleration are not mutually exclusive, and additional contributions from various $\mathrm{CR}$ accelerators may enhance the neutrino flux. Another CR source that can be associated with galaxy mergers is that the compression of the ISM gas can trigger an intense starburst. As discussed by Charbonnel et al. (2011), two processes in colliding galaxies could induce starburst: radial gas inflows can fuel a nuclear starburst, while gas turbulence and fragmentation can drive an extended starburst in clusters. Such intense star-formation can naturally lead to the injection of CRs from the ensuing massive stellar deaths, including from SNRs and HNRs. In addition, CRs may also be injected from disk-driven outflows and weak jets from AGNs (Murase et al. 2014; Tamborra et al. 2014; Liu et al. 2017). The CR contributions from these sources, which would be additional to CRs from the mergers considered here, are significantly model-dependent, and we do not attempt here a quantification of their relative importance.

One important factor that may influence the final results is the $\mathrm{CR}$ power-law index $s$, since the factor $\varepsilon^{2-s}$, the maximal CR energy as well as a new $\mathcal{C}=\left(\left(\varepsilon_{p}^{\max }\right)^{2-s}-\left(\varepsilon_{p}^{\min }\right)^{2-s}\right) /(2-s)$ are required to correct the Eq. 6 when $s$ deviates from 2.0. As presented in Eq. 6, we assume that the shock is non-radiative and infinitesimally thin and hence the Fermi first order acceleration in the strong shock limit implies $s=2$. However, a finite width of the shock can steepen the spectrum to $s \gtrsim 2.0$, while a radiative shock would produce a CR spectrum with a power-law index lower than 2.0. For radiative shocks, Blandford \& Eichler (1987)

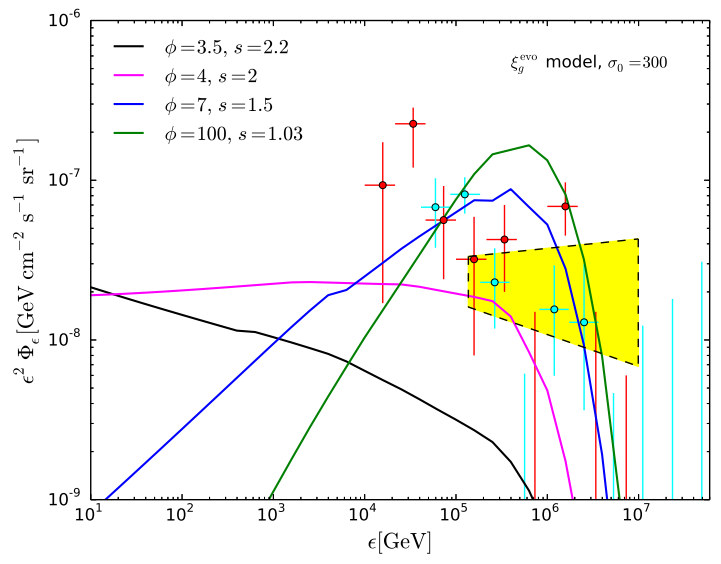

Figure 6. The neutrino fluxes for different compression ratios and $\mathrm{CR}$ power-law indices. The black, magenta, blue and greens lines correspond to the power-law indices $s=2.2,2.0,1.5$ and 1.03 .

showed that the power-law index of the accelerated CRs is $s=(\phi+2) /(\phi-1)$ where $\phi$ is the compression ratio. Moreover, Yamazaki et al. (2006) assume $\phi=7$ and $s=1.5$ as fiducial values when studying the radiative cooling of SNR shocks. Note that the compression ratio for radiative shocks with an isothermal adiabatic index $\gamma=1$ can be written as $\phi=M^{2} \gg 1$, where $M$ is the upstream Mach number. We asume thus $s=1$ in this extreme case. To illustrate how the neutrino spectra are affected by the radiative cooling and/or the width of the shocks, we plot in Fig. 6 the neutrino fluxes for four cases $s=2.2,2.0,1.5$ and 1.03 which correspond to $\phi=3.5,4,7,100$, respectively. As can be seen, a harder CR power-law index (lower $s$ ) will produce more highenergy neutrinos. Thus, in principle, a mildly radiativecooling shock $(1.5 \leq s \leq 2)$ can more easily achieve the high-energy neutrino flux in the range $10 \mathrm{TeV}$ to $\sim \mathrm{PeV}$. On the other hand, $s \gtrsim 2.1-2.2$ is disfavored because of the damping factor $\varepsilon^{2-s}$, which is consistent with previous work (Murase et al. 2013). Note that the hard spectrum is expected for the cold gas environment that would be valid in sufficiently low-mass halo mergers. If the temperature is so high, the Mach number is expected to be low, as expected for cluster mergers. In this case, the spectrum is softer for massive clusters, and details are beyond the scope of this work.

Additional contributions may arise from the galaxies moving through the cluster or dark matter halo, as their hypersonic peculiar motion will result in a shock as they plow through the intra-cluster gas, which as a result can also contribute to the diffuse $\gamma$ rays and neutrinos. Supposing as an extreme case that the loss of the galaxies' kinetic energy due to the gravitational drag is completely converted into CR energy, we estimate a CR 
energy budget of

$$
\varepsilon_{p} Q_{\varepsilon_{p}}^{(\mathrm{IGM})}=\epsilon_{p} \mathcal{C}_{\mathrm{IGM}}^{-1} \int d M_{h} \frac{4 \pi G^{2} M_{h}^{2} \rho_{c l}}{v_{s}} \frac{d N}{d M_{h}} \ln \left(\frac{R_{\mathrm{cl}}}{R_{g}}\right),
$$

which is three orders of magnitudes lower than halo mergers estimated in the previous sections, because of the tenuous intergalactic gas density. Hence, these shocks contribute only a relatively small amount of diffuse neutrinos and are negligible compared to the mergers.

\section{SUMMARY}

In summary, we found that the CR luminosity density by halo mergers can be comparable to that from starburst galaxies, which can be expected from galaxy mergers. In particular, the CR input from galaxy mergers and cluster/group mergers is comparable in the local universe, and the former is more important at higher redshifts, $z \gtrsim 2$ (see the dashed and dash-dotted lines in Figure (3). This emphasizes the importance of our results for CR reservoir models. We have considered the neutrino and $\gamma$-ray production in galaxy-galaxy and cluster/group merger environments and found that such mergers could explain a large portion of the IceCube diffuse neutrino flux. Since many more galaxy-scale, lowmass halo mergers occur at relatively high redshifts, the contribution to the diffuse $\gamma$-ray background observed by Fermi is more suppressed, due to the $\gamma \gamma$ absorption. Despite the various uncertainties due to the lack of high redshift observations of the galactic and cluster morphologies, the gas distribution and the galactic/intergalactic magnetic fields, some of the crucial and sensitive parameters including the gas fraction $\xi_{g}^{\text {evo }}$ are relatively well constrained. The parameter space left for variance of both the neutrino and $\gamma$-ray spectra is restricted by our results, as demonstrated in Figs. 4 and 5. One of the large uncertainties comes from the spectral index, and we demonstrated the cases of harder CR spectral indices, $1.5 \lesssim s \lesssim 2$, which could be expected in strong radiative shocks.

One of the predictions of the halo merger model is that the effective number density of these sources is expected to be $\sim 10^{-5} \mathrm{Mpc}^{-3}$, which is similar to the number density of starburst galaxies and AGN with disk-driven outflows. The present model is testable in the sense that such halo merger sources are detectable with nextgeneration detectors such as IceCube-Gen2 via searches for multiplets, auto-correlation, and cross-correlation signals (Murase \& Waxman 2016). One must keep in mind that the contributions from galaxy/halo mergers are degenerate with those from other possibilities, such as the starburst and AGN contributions, since a large fraction of starburst and AGN activities can be induced

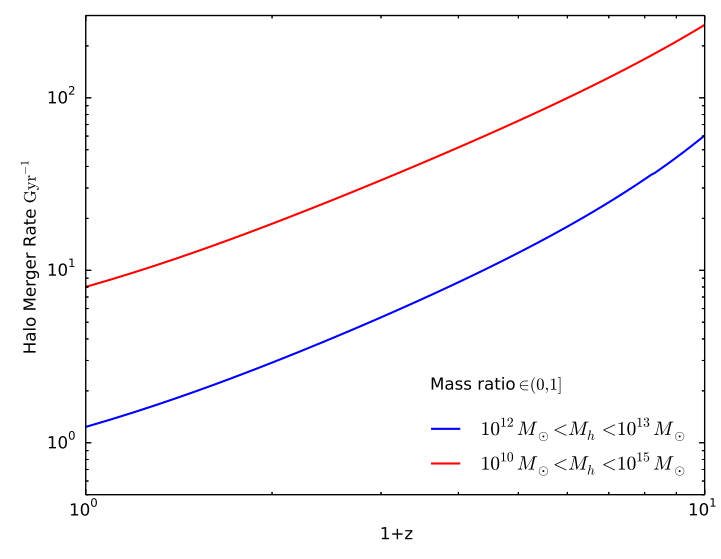

Figure 7. Merger rates from Eq. (22). The blue line represents the whole mass range $\left(10^{10} \mathrm{M}_{\odot} \sim 10^{15} \mathrm{M}_{\odot}\right)$ and the red line corresponds to $10^{12} \mathrm{M}_{\odot}<M_{h}<10^{13} \mathrm{M}_{\odot}$

by these mergers. To distinguish among these models, cross-correlation or auto-correlation studies in neutrinos and $\gamma$-rays should be useful. Also, to identify the merging sources, it will be important to investigate these sources at multi-wavelengths.

\section{ACKNOWLEDGEMENTS}

We are grateful to Zhao-Wei Zhang and Kazumi Kashiyama for useful discussions. This research was partially supported by NASA NNX13AH50G (C-C.Y., P.M.), the Alfred P. Sloan Foundation and NSF grant No. PHY-1620777 (K.M.) and NSF grant No. AST1517363 (D.J.).

\section{APPENDIX A: THE MERGER RATE}

In this section we present a comparison between our halo merger rate with the Illustris simulations (Rodriguez-Gomez et al. 2015). In our calculation, we assign a mean merger probability $P(M, z)=$ $\exp \left(-t_{\text {merger }} / t_{\text {age }}\right)$ to each dark matter halo during $t_{\text {age }}$. Here, $t_{\text {merger }}$, which can be obtained from the second equation of Eq.(5), averages all possible mass ratios, e.g. $\zeta \in(0,1]$. In our calculation, we do not need to use the cumulative merger rate over mass directly, instead the factor $\frac{d N}{d M} \frac{P(M, z)}{t_{\text {age }}}$ in the integrand of Eq. (6) is used to illustrate the number of mergers for a halo with mass $M$ and at redshift $z$. However, in order to compare our results with the simulations, it is worthwhile to estimate the average cumulative merger rate using our approach,

$$
\mathcal{R}(z)=\frac{\int \frac{d N}{d M} \frac{P(M, z)}{t_{\text {age }}} d M}{\int \frac{d N}{d M} d M} .
$$

The merger rate is shown in Fig. 7 where the blue line represents the whole mass range $\left(10^{10} \mathrm{M}_{\odot} \sim 10^{15} \mathrm{M}_{\odot}\right)$ and the red line corresponds to $10^{12} \mathrm{M}_{\odot}<M_{h}<$ 
$10^{13} \mathrm{M}_{\odot}$. In both cases, the mass ratio covers the entire interval as in the middle equation of Eq. (5) which is integrated over $\zeta$ from 0 to 1 . The merger rate given by Illustris simulations is shown in the lower panel.

One can compare our results with solid black lines in the right panel of Fig. 2 in Rodriguez-Gomez et al. (2015), since the increase in the merger rates given by simulations (as shown as colored lines) seen at low red- shifts is due to a limitation of the splitting algorithm. As can be seen, our merger rate in the same mass interval is comparable to the merger rate in the right panel with the mass ratio $\geq 1 / 1000$. Considering that we are using a totally different method and this approach is primarily designed to evaluate the merger probabilities of halos of various masses and at different redshifts, the moderate degree of discrepancy can be considered acceptable.

\section{REFERENCES}

Aartsen, M., et al. 2013a, Phys.Rev.Lett., 111, 021103

—. 2013b, Science, 342, 1242856

—. 2014, Phys.Rev.Lett., 113, 101101

Aartsen, M., Abraham, K., Ackermann, M., et al. 2015, The Astrophysical Journal, 809, 98

—. 2016, The Astrophysical Journal, 833, 3

Aartsen, M. G., et al. 2017, arXiv: 1710.01191

Abeysekara, A. U., et al. 2017a, Astrophys. J., 842, 85

—. 2017b, arXiv: 1710.10288

Ackermann, M., Ajello, M., Albert, A., et al. 2016, Physical Review Letters, 116, 151105

Ackermann, M., Ajello, M., Albert, A., et al. 2015, The Astrophysical Journal, 799, 86

Ahlers, M., \& Murase, K. 2014, Phys. Rev., D90, 023010

Alvarez-Muñiz, J., \& Mészáros, P. 2004, Physical Review D, 70, 123001

Anchordoqui, L. A., Hooper, D., Sarkar, S., \& Taylor, A. M. 2008, Astroparticle Physics, 29, 1

Anchordoqui, L. A., Paul, T. C., da Silva, L. H., Torres, D. F., \& Vlcek, B. J. 2014, Physical Review D, 89, 127304

Apel, W. D., et al. 2017, Astrophys. J., 848, 1

Baerwald, P., Bustamante, M., \& Winter, W. 2013, The Astrophysical Journal, 768, 186

Bahcall, J. N., \& Soneira, R. M. 1980, The Astrophysical Journal Supplement Series, 44, 73

Barnaby, D., \& Thronson Jr, H. A. 1992, The Astronomical Journal, 103, 41

Bechtol, K., Ahlers, M., Di Mauro, M., Ajello, M., \& Vandenbroucke, J. 2017, The Astrophysical Journal, 836, 47

Becker Tjus, J., Eichmann, B., Halzen, F., Kheirandish, A., \& Saba, S. M. 2014, Phys. Rev., D89, 123005

Behroozi, P. S., Wechsler, R. H., \& Conroy, C. 2013, The Astrophysical Journal, 770, 57

Berezinsky, V., \& Smirnov, A. Y. 1975, Astrophysics and Space Science, 32, 461

Berrington, R. C., \& Dermer, C. D. 2003, Astrophys. J., 594, 709

Blanco, C., \& Hooper, D. 2017, JCAP, 1712, 017

Blandford, R., \& Eichler, D. 1987, Physics Reports, 154, 1

Bonvin, V., Courbin, F., Suyu, S., et al. 2017, Monthly Notices of the Royal Astronomical Society, 465, 4914

Bustamante, M., Baerwald, P., Murase, K., \& Winter, W. 2014

Chakraborty, S., \& Izaguirre, I. 2015, Phys. Lett., B745, 35

Chang, X.-C., Liu, R.-Y., \& Wang, X.-Y. 2015, The Astrophysical Journal, 805, 95

Chang, X.-C., Liu, R.-Y., \& Wang, X.-Y. 2016, ApJ, 825, 148

Chang, X.-C., \& Wang, X.-Y. 2014, The Astrophysical Journal, 793,131

Charbonnel, C., Montmerle, T., \& Bournaud, F. 2011, European Astronomical Society Publications Series, 51, 107

Clowe, D., Gonzalez, A., \& Markevitch, M. 2004, The Astrophysical Journal, 604, 596

Crocker, R. M. 2012, Monthly Notices of the Royal Astronomical Society, 423, 3512
Croston, J., Pratt, G., Böhringer, H., et al. 2008, Astronomy \& Astrophysics, 487, 431

Davis, M., \& Peebles, P. 1977, The Astrophysical Journal Supplement Series, 34, 425

—. 1983, The Astrophysical Journal, 267, 465

Denton, P. B., \& Tamborra, I. 2017, arXiv: 1711.00470

Dermer, C. D., Murase, K., \& Inoue, Y. 2014, Journal of High Energy Astrophysics, 3, 29

Drury, L. O. 1983, Reports on Progress in Physics, 46, 973

Eke, V. R., Navarro, J. F., \& Frenk, C. S. 1998, The Astrophysical Journal, 503, 569

Fakhouri, O., Ma, C.-P., \& Boylan-Kolchin, M. 2010, Monthly Notices of the Royal Astronomical Society, 406, 2267

Fang, K., \& Murase, K. 2018, Nature Physics, 1

Fialkov, A., \& Loeb, A. 2016, arXiv preprint arXiv:1611.01386

Finke, J. D., Razzaque, S., \& Dermer, C. D. 2010, The Astrophysical Journal, 712, 238

Fujita, Y., \& Sarazin, C. L. 2001, The Astrophysical Journal, 563,660

Gaisser, T., \& Halzen, F. 2014, Annual Review of Nuclear and Particle Science, 64, 101

Groth, E. J., \& Peebles, P. 1977, The Astrophysical Journal, 217, 385

Gupta, N., \& Zhang, B. 2007, Astroparticle Physics, 27, 386

Halzen, F. 2016, Nature Phys., 13, 232

Halzen, F., \& Zas, E. 1997, Astrophys. J., 488, 669

Hamilton, A., Kumar, P., Lu, E., \& Matthews, A. 1991, The Astrophysical Journal, 374, L1

Hawkins, E., Maddox, S., Cole, S., et al. 2003, Monthly Notices of the Royal Astronomical Society, 346, 78

Inoue, Y., Inoue, S., Kobayashi, M. A., et al. 2013, The Astrophysical Journal, 768, 197

Jenkins, A., Frenk, C., White, S. D., et al. 2001, Monthly Notices of the Royal Astronomical Society, 321, 372

Jing, Y., Mo, H., \& Börner, G. 1998, The Astrophysical Journal, 494, 1

Kafexhiu, E., Aharonian, F., Taylor, A. M., \& Vila, G. S. 2014, Physical Review D, 90, 123014

Kashiyama, K., \& Mészáros, P. 2014, The Astrophysical Journal Letters, 790, L14

Keeney, B. A., Danforth, C. W., Stocke, J. T., et al. 2006, The Astrophysical Journal, 646, 951

Kimura, S. S., Murase, K., \& Toma, K. 2015, Astrophys. J., 806, 159

Kylafis, N. D., \& Bahcall, J. N. 1987, The Astrophysical Journal, 317, 637

Lamastra, A., Menci, N., Fiore, F., et al. 2017, Astron. Astrophys., 607, A18

Lewis, A., Challinor, A., \& Lasenby, A. 2000, Astrophys. J., 538, 473

Lisanti, M., Mishra-Sharma, S., Necib, L., \& Safdi, B. R. 2016, Astrophys. J., 832, 117 
Liu, R.-Y., Murase, K., Inoue, S., Ge, C., \& Wang, X.-Y. 2017, arXiv: 1712.10168

Loeb, A., \& Waxman, E. 2006, Journal of Cosmology and Astroparticle Physics, 2006, 003

Mannheim, K. 1995, Astropart. Phys., 3, 295

Markevitch, M., Gonzalez, A., Clowe, D., et al. 2004, The Astrophysical Journal, 606, 819

Meszaros, P., \& Waxman, E. 2001, Physical Review Letters, 87, 171102

Murase, K. 2008, Physical Review D, 78, 101302

Murase, K., Ahlers, M., \& Lacki, B. C. 2013, Physical Review D, 88,121301

Murase, K., \& Beacom, J. F. 2012, Journal of Cosmology and Astroparticle Physics, 2012, 043

Murase, K., Guetta, D., \& Ahlers, M. 2016, Phys. Rev. Lett., 116, 071101

Murase, K., Inoue, S., \& Nagataki, S. 2008, The Astrophysical Journal Letters, 689, L105

Murase, K., Inoue, Y., \& Dermer, C. D. 2014, Physical Review D, 90, 023007

Murase, K., \& Ioka, K. 2013, Physical Review Letters, 111, 121102

Murase, K., Ioka, K., Nagataki, S., \& Nakamura, T. 2006, The Astrophysical Journal Letters, 651, L5

Murase, K., \& Waxman, E. 2016, Phys. Rev., D94, 103006

Murray, S., Power, C., \& Robotham, A. 2013, Monthly Notices of the Royal Astronomical Society: Letters, 434, L61

Padovani, P., Petropoulou, M., Giommi, P., \& Resconi, E. 2015, Mon. Not. Roy. Astron. Soc., 452, 1877

Peacock, J., \& Dodds, S. 1996, Monthly Notices of the Royal Astronomical Society, 280, L19

Peebles, P. J. 1974, The Astrophysical Journal, 189, L51

Peebles, P. J. E. 1980, The large-scale structure of the universe (Princeton university press)

Petropoulou, M., Dimitrakoudis, S., Padovani, P., Mastichiadis, A., \& Resconi, E. 2015, Monthly Notices of the Royal Astronomical Society, 448, 2412
Press, W. H., \& Schechter, P. 1974, The Astrophysical Journal, 187,425

Reed, D. S., Bower, R., Frenk, C. S., Jenkins, A., \& Theuns, T. 2006, Monthly Notices of the Royal Astronomical Society, 374, 2

Rodriguez-Gomez, V., Genel, S., Vogelsberger, M., et al. 2015, Monthly Notices of the Royal Astronomical Society, 449, 49

Sargent, M. T., Daddi, E., Béthermin, M., et al. 2014, The Astrophysical Journal, 793, 19

Senno, N., Mészáros, P., Murase, K., Baerwald, P., \& Rees, M. J. 2015, The Astrophysical Journal, 806, 24

Senno, N., Murase, K., \& Mészáros, P. 2016, Physical Review D, 93, 083003

Sheth, R. K., \& Tormen, G. 1999, Monthly Notices of the Royal Astronomical Society, 308, 119

Shibuya, T., Ouchi, M., \& Harikane, Y. 2015, The Astrophysical Journal Supplement Series, 219, 15

Smith, R. E., Peacock, J. A., Jenkins, A., et al. 2003, Monthly Notices of the Royal Astronomical Society, 341, 1311

Stecker, F. W. 2013, Physical Review D, 88, 047301

Stecker, F. W., Done, C., Salamon, M. H., \& Sommers, P. 1991, Physical Review Letters, 66, 2697

Tamborra, I., \& Ando, S. 2016, Physical Review D, 93, 053010

Tamborra, I., Ando, S., \& Murase, K. 2014, Journal of Cosmology and Astroparticle Physics, 2014, 043

Van Der Kruit, P., \& Searle, L. 1981, Astronomy and Astrophysics, 95, 116

Wang, X.-Y., \& Dai, Z.-G. 2009, The Astrophysical Journal Letters, 691, L67

Waxman, E., \& Bahcall, J. 1997, Physical Review Letters, 78, 2292

Xiao, D., \& Dai, Z. 2014, The Astrophysical Journal, 790, 59

—. 2015, The Astrophysical Journal, 805, 137

Xiao, D., Mészáros, P., Murase, K., \& Dai, Z.-G. 2016, ApJ, 826, 133

Yamazaki, R., Kohri, K., Bamba, A., et al. 2006, Monthly Notices of the Royal Astronomical Society, 371, 1975

Zehavi, I., Blanton, M. R., Frieman, J. A., et al. 2002, The Astrophysical Journal, 571, 172 